\title{
EFFECT OF SUSPENDED PARTICLES ON THERMAL CONVECTION IN RIVLIN-ERICKSEN FLUID IN A DARCY-BRINKMAN POROUS MEDIUM
}

\author{
G.C. Rana $^{1}$ and R.C. Thakur ${ }^{2}$ \\ ${ }^{1}$ Department of Mathematics \\ NSCBM Govt. P. G. College, Hamirpur-177 005, Himachal Pradesh \\ INDIA Email: drgcrana15@gmail.com \\ ${ }^{2}$ Department of Mathematics \\ Government P. G. College Dhaliara- 177103 Himachal Pradesh \\ INDIA Email: rameshnahan@yahoo.com
}

\begin{abstract}
In this paper, the effect of suspended particles on thermal convection in an incompressible Rivlin-Ericksen elastico-viscous fluid in a porous medium is considered. For the porous medium, the Brinkman model is employed. By applying a normal mode analysis method, the dispersion relation has been derived and solved analytically. It is observed that the medium permeability, suspended particles, gravity field and viscoelasticity introduce oscillatory modes. For stationary convection, it is observed that the Darcy number has a stabilising effect, whereas the suspended particles and medium permeability have destabilising effects on the system. The effects of suspended particles, the Darcy number and the medium permeability have been presented graphically to depict the stability characteristics, which are in good agreement with the results derived analytically.
\end{abstract}

Keywords: Brinkman porous medium, Rivlin-Ericksen fluid, suspended particles, thermal convection, viscosity, viscoelasticity.

\section{INTRODUCTION}

In recent years, considerable interest has been shown in the study of thermal instability in a porous medium, because it has various applications in geophysics, food processing and nuclear reactors (Rana 2011). Chandrasekhar (1981) gave a detailed account of the thermal instability of a Newtonian fluid under varying assumptions of hydrodynamics and hydromagnetics. Lapwood (1948) studied the convective flow in a porous medium using linearised stability theory. The Rayleigh instability of a thermal boundary layer in a flow through a porous medium has been considered by Wooding (1960). Scanlon and Segel (1973) considered the effect of suspended particles on the onset of Be'nard convection and found that the critical Rayleigh number was reduced solely because the heat capacity of the pure gas was supplemented by the particles. Sharma and Sharma (1991) have studied the stability of stratified fluids in a porous medium in the presence of suspended particles and a variable magnetic field; the suspended particles were found to destabilise the layer. Sharma and Sunil (1994) have studied the thermal instability of an Oldroydian viscoelastic fluid with suspended particles in hydromagnetics in a porous medium. There are many elastico-viscous fluids that cannot be characterised by either Maxwell's or Oldroyd's constitutive relations. One such class of elastico-viscous fluid is a Rivlin-Ericksen fluid. Rivlin and Ericksen (1955) proposed a theoretical model for such an elastico-viscous fluid. Rana and Kumar (2010) studied the effect of suspended 
particles on the thermal instability of a Rivlin-Ericksen elastico-viscous rotating fluid in a porous medium. Rana (2012) studied the thermal instability of a compressible RivlinEricksen elastico-viscous rotating fluid permeated with suspended dust particles in porous medium.

The investigation in porous media began with the simple Darcy model and was extended gradually to the Darcy-Brinkman model. A good account of the convection problems in a porous medium is given by Vafai and Hadim (2000), Satya Narayana, Ramireddy and Venkataramana (2011), Ingham and Pop (1981) and Nield and Bejan (2006). Kuznetsov and Nield (2010) have studied the thermal instability in a porous medium layer saturated by a nanofluid in a Brinkman porous medium. Sharma, Kishore \& Rana (2001) have studied the instability of streaming Rivlin-Ericksen fluids in a porous medium. Recently, Rana and Thakur (2012) studied the thermal instability of couple-stress fluid permeated with suspended particles saturating a porous medium. The interest for investigations of non-Newtonian fluids is also motivated by a wide range of engineering applications, which include ground pollution by chemicals that are nonNewtonian, such as lubricants and polymers and in the treatment of sewage sludge in drying beds. Recently, polymers have been used in agriculture, communications appliances and in bio-medical applications. Examples of these applications are filtration processes, packed bed reactors, insulation systems, ceramic processing, enhanced oil recovery and chromatography, etc. Bearing in mind the importance of the various applications mentioned above, the objective of the present paper is to study the effect of suspended particles on thermal convection in a Rivlin-Ericksen elastico-viscous fluid in a Brinkman porous medium. This necessitates an additional parameter, namely the Darcy number.

\section{MATHEMATICAL MODEL AND PERTURBATION EQUATIONS}

Consider an infinite, horizontal, incompressible Rivlin-Ericksen elastico-viscous fluid of depth $d$, bounded by the planes $z=0$ and $z=d$ in an isotropic and homogeneous medium of porosity $\varepsilon$ and permeability $k_{1}$, which is acted upon by gravity $\mathbf{g}(0,0,-\mathrm{g})$. This layer is heated from below, such that a steady adverse temperature gradient $\beta(=|d T / d z|)$ is maintained. The character of equilibrium of this initial static state is determined by supposing that the system is slightly disturbed and then by following its further evolution.

Let $\rho, v, v^{\prime}, p, \varepsilon, T, \alpha, \tilde{\mu}$ and $v(0,0,0)$, denote the density, kinematic viscosity, kinematic viscoelasticity, pressure, medium porosity, temperature, thermal coefficient of expansion, effective viscosity of pure fluid and velocity of the fluid, respectively.

The conservation of momentum, mass, temperature and equation of state for a Rivlin-Ericksen elastico-viscous fluid in a Brinkman porous medium are expressed in Eqs (1)-(4), respectively.

$$
\begin{gathered}
\frac{1}{\varepsilon}\left[\frac{\partial v}{\partial t}+\frac{1}{\varepsilon}(\boldsymbol{v} \cdot \nabla) \boldsymbol{v}\right]=-\frac{1}{\rho_{0}} \nabla p+\boldsymbol{g}\left(1+\frac{\delta \rho}{\rho_{0}}\right)-\frac{1}{k_{1}}\left(v+v^{\prime} \frac{\partial}{\partial t}\right) \boldsymbol{v}+\frac{\widetilde{\mu}}{\rho_{0}} \nabla^{2} \boldsymbol{v}+\frac{K^{\prime} N}{\rho_{0} \varepsilon}\left(\boldsymbol{v}_{\boldsymbol{d}}-\boldsymbol{v}\right) \\
\nabla \cdot \boldsymbol{v}=0 \\
E \frac{\partial T}{\partial t}+(\boldsymbol{v} \cdot \nabla) T+\frac{m N C_{p t}}{\rho_{0} C_{f}}\left[\varepsilon \frac{\partial}{\partial t}+v_{d} \cdot \nabla\right] T=\kappa \nabla^{2} \mathrm{~T} \\
\rho=\rho_{0}\left[1-\alpha\left(T-T_{0}\right)\right]
\end{gathered}
$$

where the suffix zero refers to values at the reference level $z=0$. 


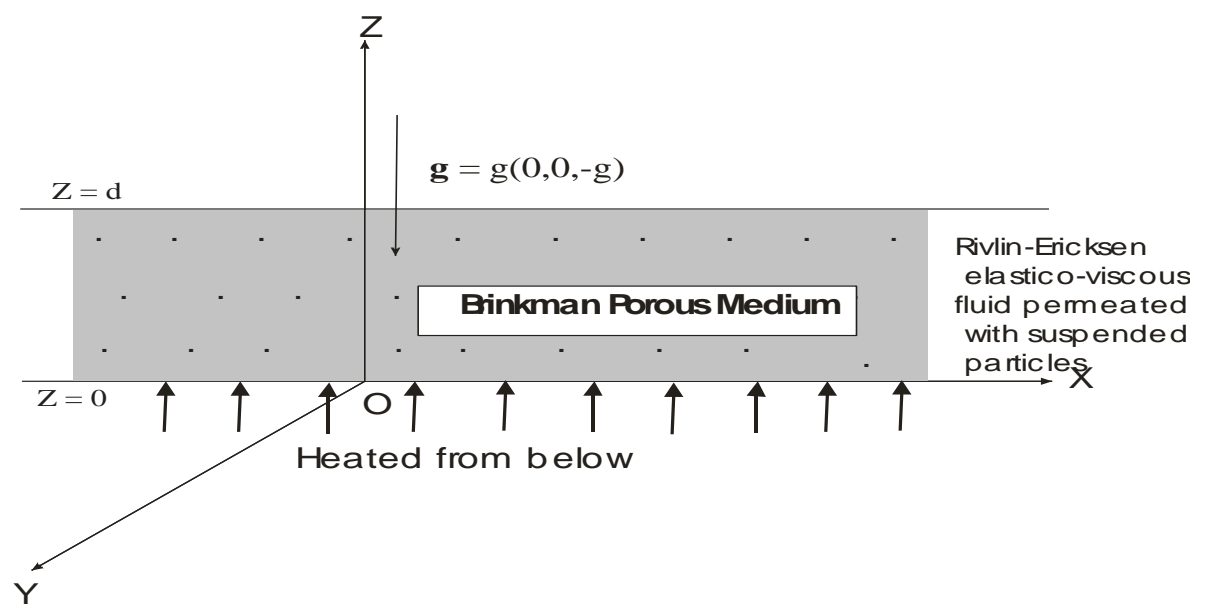

Figure 1. Schematic sketch of physical situation.

Here $v_{d}(\bar{x}, t)$ and $N(\bar{x}, t)$ denote the velocity and number density of the particles, respectively, $K^{\prime}=6 \pi \eta \rho v$, where $\eta$ is the particle radius, $K^{\prime}$ is the Stokes drag coefficient, $v_{d}=(l, r, s)$ and $\bar{x}=(x, y, z)$.

$$
E=\varepsilon+(1-\varepsilon)\left(\frac{\rho_{s} c_{s}}{\rho_{0} c_{f}}\right)
$$

which is constant, $\kappa$ is the thermal diffusivity, $\rho_{s}, c_{s} ; \rho_{0}, c_{f}$ denote the density and heat capacity of solid (porous) matrix and fluid, respectively. If $m N$ is the mass of particles per unit volume, then the equations of motion and continuity for the particles are expressed in Eq. (5) and Eq. (6), respectively.

$$
\begin{aligned}
& m N\left[\frac{\partial v_{d}}{\partial t}+\frac{1}{\varepsilon}\left(v_{d} \cdot \nabla\right) v_{d}\right]=K^{\prime} N\left(v-v_{d}\right) \\
& \varepsilon \frac{\partial N}{\partial t}+\nabla \cdot\left(N v_{d}\right)=0
\end{aligned}
$$

The presence of particles adds an extra force term proportional to the velocity difference between the particles and the fluid and appears in the equation of motion (Eq. (1)). Because the force exerted by the fluid on the particles is equal and opposite to that exerted by the particles on the fluid, there must be an extra force term, equal in magnitude but opposite in sign, in the equations of motion for the particles (Eq. (6)). The buoyancy force on the particles is neglected. Inter-particle reactions are not considered either because the distance between the particles is quite large compared with their diameters. These assumptions have been used in writing the equations of motion (Eq. (6)) for the particles. The initial state of the system is taken to be a quiescent layer (no settling) with a uniform particle distribution number. The initial state is defined as Eq. (7).

$$
\boldsymbol{v}=(0,0,0), T=-\beta z+T_{0}, \rho=\rho_{0}(1+\alpha \beta z)
$$

This is an exact solution to the governing equations. Let $\boldsymbol{v}(\mathrm{u}, \mathrm{v}, \mathrm{w}), \theta, \delta p$ and $\delta \rho$ denote the perturbations in the fluid velocity $v(0,0,0)$, temperature $T$, pressure $p$ and density $\rho$, respectively. The change in density $\delta \rho$ caused by perturbation $\theta$ in temperature is given by Eq. (8). 


$$
\delta \rho=-\alpha \rho_{0} \theta
$$

The linearised perturbation equations governing the motion of the fluid are as follows:

$$
\begin{gathered}
\frac{1}{\varepsilon} \frac{\partial v}{\partial t}=-\frac{1}{\rho_{0}} \nabla \delta p-g \frac{\delta \rho}{\rho_{0}}-\frac{1}{k_{1}}\left(v+v^{\prime} \frac{\partial}{\partial t}\right) \boldsymbol{v}+\frac{\widetilde{\mu}}{\rho_{0}} \nabla^{2} \boldsymbol{v}+\frac{K^{\prime} N}{\rho_{0} \epsilon}\left(\boldsymbol{v}_{\boldsymbol{d}}-\boldsymbol{v}\right) \\
\nabla \cdot \boldsymbol{v}=0 \\
(E+b \varepsilon) \frac{\partial \theta}{\partial t}=\beta(w+b s)+\kappa \nabla^{2} \theta
\end{gathered}
$$

Where $b=\frac{m N C_{p t}}{\rho_{0} C_{f}}$ and $w, s$ are the vertical fluid and particles velocity.

In Cartesian form, Eqs (9)-(11) with the help of Eq. (8) can be expressed as Eq. (12) and Eq. (13).

$$
\begin{gathered}
\frac{1}{\varepsilon} \frac{\partial u}{\partial t}=-\frac{1}{\rho_{0}} \frac{\partial}{\partial x}(\delta p)-\frac{1}{k_{1}}\left(v+v^{\prime} \frac{\partial}{\partial t}\right) u+\frac{\widetilde{\mu}}{\rho_{0}} \nabla^{2} u-\frac{m N}{\epsilon\left(\frac{m \partial}{K^{\prime} \partial t}+1\right) \rho_{0}} \frac{\partial u}{\partial t} \\
\frac{1}{\varepsilon} \frac{\partial v}{\partial t}=-\frac{1}{\rho_{0}} \frac{\partial}{\partial y}(\delta p)-\frac{1}{k_{1}}\left(v+v^{\prime} \frac{\partial}{\partial t}\right) v+\frac{\widetilde{\mu}}{\rho_{0}} \nabla^{2} v-\frac{m N}{\epsilon\left(\frac{m \partial}{K^{\prime} \partial t}+1\right) \rho_{0}} \frac{\partial v}{\partial t} \\
\frac{1}{\varepsilon} \frac{\partial w}{\partial t}=-\frac{1}{\rho_{0}} \frac{\partial}{\partial z}(\delta p)+g \alpha \theta-\frac{1}{k_{1}}\left(v+v^{\prime} \frac{\partial}{\partial t}\right) w+\frac{\widetilde{\mu}}{\rho_{0}} \nabla^{2} w-\frac{m N}{\epsilon\left(\frac{m \partial}{K^{\prime} \partial t}+1\right) \rho_{0}} \frac{\partial w}{\partial t} \\
\frac{\partial u}{\partial x}+\frac{\partial v}{\partial y}+\frac{\partial w}{\partial z}=0 \\
(E+b \varepsilon) \frac{\partial \theta}{\partial t}=\beta(w+b s)+\kappa \nabla^{2} \theta
\end{gathered}
$$

Operating Eqs (12) and (13) by $\frac{\partial}{\partial x}$ and $\frac{\partial}{\partial y}$, respectively and adding and using Eq. (8), we get:

$$
\frac{1}{\varepsilon} \frac{\partial}{\partial t}\left(\frac{\partial w}{\partial z}\right)=\frac{1}{\rho_{0}}\left(\nabla^{2}-\frac{\partial^{2}}{\partial z^{2}}\right) \delta p-\frac{1}{k_{1}}\left(v+v^{\prime} \frac{\partial}{\partial t}\right)\left(\frac{\partial w}{\partial z}\right)+\frac{\widetilde{\mu}}{\rho_{0}} \nabla^{2}\left(\frac{\partial w}{\partial z}\right)-\frac{m N}{\epsilon\left(\frac{m \partial}{K^{\prime} \partial t}+1\right) \rho_{0}} \frac{\partial}{\partial t}\left(\frac{\partial w}{\partial z}\right)
$$

Operating Eqs (14) and (17) by $\left(\nabla^{2}-\frac{\partial^{2}}{\partial z^{2}}\right)$ and $\frac{\partial}{\partial z}$, respectively and adding to eliminate $\delta p$ between Eqs (14) and (17), we get:

$$
\frac{1}{\varepsilon} \frac{\partial}{\partial t}\left(\nabla^{2} \mathrm{w}\right)=-\frac{1}{k_{1}}\left(v+v^{\prime} \frac{\partial}{\partial t}\right) \nabla^{2} \mathrm{w}+\frac{\widetilde{\mu}}{\rho_{0}} \nabla^{4} \mathrm{w}+\mathrm{g}\left(\frac{\partial^{2}}{\partial x^{2}}+\frac{\partial^{2}}{\partial y^{2}}\right) \alpha \theta-\frac{m N}{\epsilon\left(\frac{m \partial}{K^{\prime} \partial t}+1\right) \rho_{0}} \frac{\partial}{\partial t}\left(\nabla^{2} \mathrm{w}\right)
$$

Where $\nabla^{2}=\frac{\partial^{2}}{\partial x^{2}}+\frac{\partial^{2}}{\partial y^{2}}+\frac{\partial^{2}}{\partial z^{2}}$

\section{THE DISPERSION RELATION}

Following the normal mode analyses, we assume that the perturbation quantities have $x$, $y$ and $t$ dependence of the form:

$$
[w, \theta]=[W(z), \Theta(z)] \exp (i l x+i m y+n t)
$$


where $l$ and $m$ are the wave numbers in the $x$ and $y$ directions, $k=\left(l^{2}+m^{2}\right)^{1 / 2}$ is the resultant wave number and $n$ is the frequency of the harmonic disturbance, which is a complex constant.

Using Eq. (19) in Eqs (18) and (16) becomes:

$$
\begin{gathered}
\frac{n}{\varepsilon}\left[\frac{d^{2}}{d z^{2}}-k^{2}\right] W=-g k^{2} \alpha \Theta-\frac{1}{k_{1}}\left(v+v^{\prime} n\right)\left(\frac{d^{2}}{d z^{2}}-k^{2}\right) W+\frac{\widetilde{u}}{\rho_{0}}\left(\frac{d^{2}}{d z^{2}}-k^{2}\right)^{2} W-\frac{m N}{\epsilon\left(\frac{m \partial}{k^{\prime} \partial t}+1\right) \rho_{0}}\left(\frac{d^{2}}{d z^{2}}-k^{2}\right) W \\
(E+b \varepsilon) \frac{\partial \theta}{\partial t}=\beta(w+b s)+\kappa\left(\frac{d^{2}}{d z^{2}}-k^{2}\right) \Theta
\end{gathered}
$$

Eqs (20) and (21) in non-dimensional form, become:

$$
\begin{gathered}
{\left[1+\left(\frac{P_{l}}{\varepsilon}+\frac{M P_{l}}{\varepsilon\left(1+\tau_{1} \sigma\right)}+F\right) \sigma-D_{A}\left(D^{2}-a^{2}\right)\right]\left(D^{2}-a^{2}\right) W+\frac{g a^{2} d^{2} P_{l} \alpha \Theta}{v}=0} \\
{\left[D^{2}-a^{2}-E_{1} p_{1} \sigma\right] \Theta=-\frac{\beta d^{2}}{\kappa}\left(\frac{B+\tau_{1} \sigma}{1+\tau_{1} \sigma}\right) W}
\end{gathered}
$$

where $a=k d, \sigma=\frac{n d^{2}}{v}, F=\frac{v^{\prime}}{d^{2}}$ and $P_{l}=\frac{k_{1}}{d^{2}}$ is the dimensionless medium permeability, $p_{1}=\frac{v}{\kappa}$ is the thermal Prandtl number and $D_{A}=\frac{\widetilde{\mu} k_{1}}{\mu d^{2}}$ is the Darcy number modified by the viscosity ratio.

Eliminating $\Theta$ between Eqs (22) and (23), we obtain:

$$
\left[1+\left(\frac{P_{l}}{\varepsilon}+\frac{M P_{l}}{\varepsilon\left(1+\tau_{1} \sigma\right)}+F\right) \sigma-D_{A}\left(D^{2}-a^{2}\right)\right]\left(D^{2}-a^{2}\right)\left(D^{2}-a^{2}-E p_{1} \sigma\right) W-R a^{2} P_{l}\left(\frac{B+\tau_{1} \sigma}{1+\tau_{1} \sigma}\right) W=0
$$

where $R=\frac{g \alpha \beta d^{4}}{v \kappa}$ is the thermal Rayleigh number.

Assume that the temperature at the boundaries remains fixed, the fluid layer is confined between two boundaries and that the adjoining medium is electrically nonconducting. The boundary conditions appropriate to the problem are (Chandrasekhar, 1981):

$$
W=D^{2} W=D^{4} W=\Theta=0 \text { at } z=0 \text { and } 1
$$

The case of two free boundaries, although a little artificial, is the most appropriate for stellar atmospheres. Using the boundary conditions (Eq. (25)), all the even order derivatives of $W$ must vanish for $z=0$ and $z=1$ and hence, the proper solution of $W$ characterising the lowest mode is:

$$
W=W_{0} \sin \pi z ; W_{0} \text { is a constant }
$$

Substituting Eq. (27) in Eq. (25), we get:

$$
R_{1} x P=\left[1+\left(\frac{P}{\varepsilon}+\frac{M P}{\varepsilon\left(1+\tau_{1} i \sigma_{1}\right)}+\pi^{2} F\right) i \sigma_{1}+D_{A_{1}}(1+x)\right](1+x)\left(1+x+E_{1} p_{1} i \sigma_{1}\right)\left(\frac{1+\tau_{1} \pi^{2} i \sigma_{1}}{B+\tau_{1} \pi^{2} i \sigma_{1}}\right)
$$


where $R_{1}=\frac{R}{\pi^{4}}, T_{A_{1}}=\frac{T_{A}}{\pi^{4}}, D_{A_{1}}=\frac{D_{A}}{\pi^{2}}, x=\frac{a^{2}}{\pi^{2}}, i \sigma_{1}=\frac{\sigma}{\pi^{2}}, P=\pi^{2} P_{l}, \tau=\frac{m}{K^{\prime}}, \tau_{1}=\frac{\tau v}{d^{2}}$, $M=\frac{m N}{\rho_{0}}, E_{1}=E+b \varepsilon, \mathrm{B}=\mathrm{b}+1$.

Eq. (27) is the required dispersion relation accounting for the onset of thermal convection in a Rivlin-Ericksen elastico-viscous fluid permeated with suspended particles in a Brinkman porous medium.

\section{STABILITY OF THE SYSTEM AND OSCILLATORY MODES}

The possibility of oscillatory modes in a Rivlin-Ericksen elastico-viscous fluid due to the presence of suspended particles, viscoelasticity, medium permeability and gravity field will be examined. Multiply Eq. (22) by $W^{*}$ the complex conjugate of $W$, integrating over the range of $z$ and making use of Eq. (23) with the help of boundary conditions (Eq. (25)), we obtain:

$$
\left[1+\left(\frac{P_{l}}{\varepsilon}+\frac{M P_{l}}{\varepsilon\left(1+\tau_{1} \sigma\right)}+F\right) \sigma\right] I_{1}-D_{A} I_{2}-\frac{g a^{2} \alpha \kappa P_{l}}{v \beta}\left(\frac{1+\tau_{1} \sigma^{*}}{B+\tau_{1} \sigma^{*}}\right)\left(I_{3}+E p_{1} \sigma^{*} I_{4}\right)=0
$$

where

$$
\begin{aligned}
& I_{1}=\int_{0}^{1}\left(|D W|^{2}+a^{2}|W|^{2}\right) d z, \\
& I_{2}=\int_{0}^{1}\left(|D W|^{4}+2 a^{2}|D W|^{2}+a^{4}|W|^{2}\right) d z, \\
& I_{3}=\int_{0}^{1}\left(|D \Theta|^{2}+a^{2}|\Theta|^{2}\right) d z, \\
& I_{4}=\int_{0}^{1}|\Theta|^{2} d z,
\end{aligned}
$$

The integral part $I_{1}-I_{4}$ are all positive definite. Putting $\sigma=i \sigma_{i}$ in Eq. (28), where $\sigma_{i}$ is real and equating the imaginary parts, we obtain:

$$
\sigma_{i}\left[\left(\frac{P_{l}}{\varepsilon}+\frac{M P_{l}}{\varepsilon\left(1+\tau_{1}^{2} \sigma_{i}^{2}\right)}+F\right) I_{1}+\frac{g a^{2} \alpha \kappa P_{l}}{v \beta}\left\{\left(\frac{\tau_{1}(B-1)}{B^{2}+\tau_{1}^{2} \sigma_{i}^{2}}\right) I_{3}+\left(\frac{\tau_{1}(B-1)}{B^{2}+\tau_{1}^{2} \sigma_{i}^{2}}\right) E_{1} p_{1} I_{4}\right\}\right]=0
$$

Eq. (29) implies that $\sigma_{i}=0$ or $\sigma_{i} \neq 0$, which means that the modes may be nonoscillatory or oscillatory. The oscillatory modes are introduced due to the presence of viscosity, viscoelasticity, suspended particles and medium permeability, which were non-existent in their absence.

\section{THE STATIONARY CONVECTION}

For stationary convection, putting $\sigma=0$ in Eq. (27), we obtain:

$$
R_{1}=\frac{(1+x)^{2}}{x P B}\left[1+(1+x) D_{A_{1}}\right]
$$

Eq. (30) expresses the modified Rayleigh number $R_{1}$ as a function of the dimensionless wave number $x$ and the parameters $B, D_{A_{1}}$ and $P$ and the Rivlin-Ericksen elastico-viscous fluid behaves like an ordinary Newtonian fluid because the elasticoviscous parameter $F$ vanishes with $\sigma$. To study the effects of suspended particles, the 
Darcy number and medium permeability, the behaviour of $\frac{d R_{1}}{d B}, \frac{d R_{1}}{d D_{A_{1}}}$ and $\frac{d R_{1}}{d P}$ has been examined analytically. From Eq. (30), we get:

$$
\frac{d R_{1}}{d B}=-\left(\frac{1+x}{x P B^{2}}\right)\left[1+(1+x) D_{A_{1}}\right]
$$

which is negative. Hence, the suspended particles have a destabilising effect on the thermal convection in a Rivlin-Ericksen elastico-viscous fluid in a Brinkman porous medium. This destabilising effect is in agreement with earlier work (Scanlon \& Segel 1973; Sharma \& Sharma, 1991; Sharma \& Sunil, 1994; Rana \& Kumar, 2010; Rana, 2012).

From Eq. (30), we get:

$$
\frac{d R_{1}}{d D_{A_{1}}}=\frac{(1+x)^{3}}{x P B}
$$

which is positive, implying thereby the stabilising effect of the Darcy number on the thermal convection in a Rivlin-Ericksen elastico-viscous fluid permeated with suspended particles in a Brinkman porous medium. This stabilising effect of the Darcy number is in agreement with the earlier work of Rana (2011).

It is evident from Eq. (31) that:

$$
\frac{d R_{1}}{d P}=-\frac{(1+x)^{2}}{x B P^{2}}\left[1+(1+x) D_{A_{1}}\right]
$$

From Eq. (33), it is observed that medium permeability has a destabilising effect on the thermal convection in a Rivlin-Ericksen elastico-viscous fluid permeated with suspended particles in a Brinkman porous medium. This destabilising effect is in agreement with earlier work (Scanlon \& Segel, 1973; Sharma \& Sharma, 1991; Sharma \& Sunil; 1994, Rana \& Kumar, 2010; Rana, 2012).

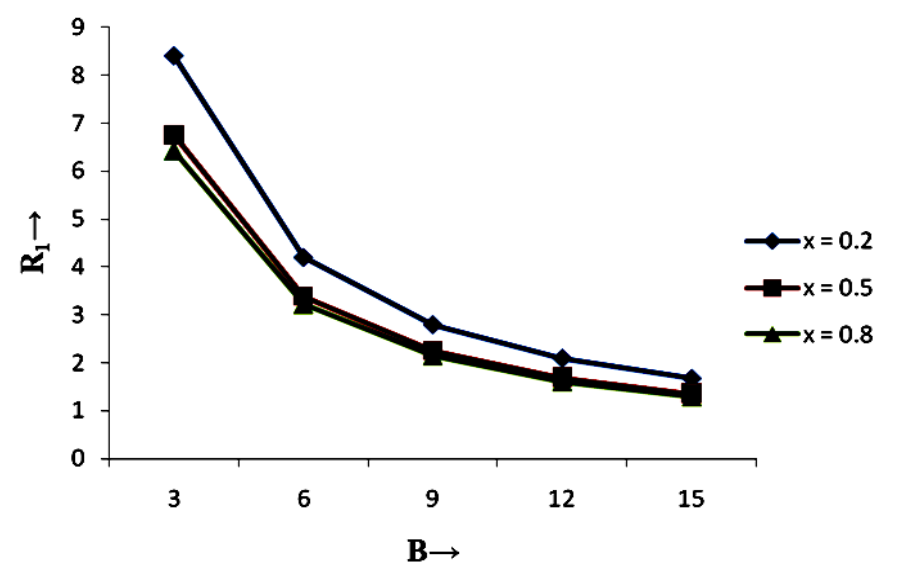

Figure 2. Variation of Rayleigh number $R_{1}$ with suspended particles $\mathrm{B}$ for $P=2$ and $D_{A_{1}}=10$ for fixed wave numbers $x=0.2, x=0.5$ and $x=0.8$.

The dispersion relation in Eq. (30) is analysed numerically to depict the stability characteristics. In Figure 2, the Rayleigh number $R_{1}$ is plotted against suspended particles $B$ for $P=2$ and $D_{A_{1}}=10$ for fixed wave numbers $x=0.2, x=0.5$ and $x=0.8$. 
This shows that the suspended particles have a destabilising effect on the thermal instability of a Rivlin-Ericksen elastico-viscous fluid in a Brinkman porous medium for fixed wave numbers $x=0.2, x=0.5$ and $x=0.8$, which clearly verifies the results numerically, as derived in Eq. (31). In Figure 3, the Rayleigh number $R_{1}$ is plotted against the Darcy number $D_{A_{1}}$ for $P=2, B=3$ for fixed wave numbers $\mathrm{x}=0.2, x=0.5$ and $\mathrm{x}=0.8$. This shows that the Darcy number has a stabilising effect on the thermal convection in a Rivlin-Ericksen elastico-viscous fluid permeated with suspended particles in a Brinkman porous medium, which clearly verifies the results numerically, as derived in equation (32). In Figure 4, the Rayleigh number $R_{1}$ is plotted against medium permeability $P$ for $D_{A_{1}}=10$ and $B=3$ for fixed wave numbers $\mathrm{x}=0.2, x=0.5$ and $\mathrm{x}=0.8$. This shows that medium permeability has a destabilising effect on the thermal convection in a Rivlin-Ericksen elastico-viscous fluid permeated with suspended particles in a Brinkman porous medium, which clearly verifies the results numerically, as derived in Eq. (33).



Figure 3. Variation of Rayleigh number $R_{1}$ with Darcy number $D_{A_{1}}$ for $P=2$ and $B=3$ for fixed wave numbers $x=0.2, x=0.5$ and $x=0.8$.

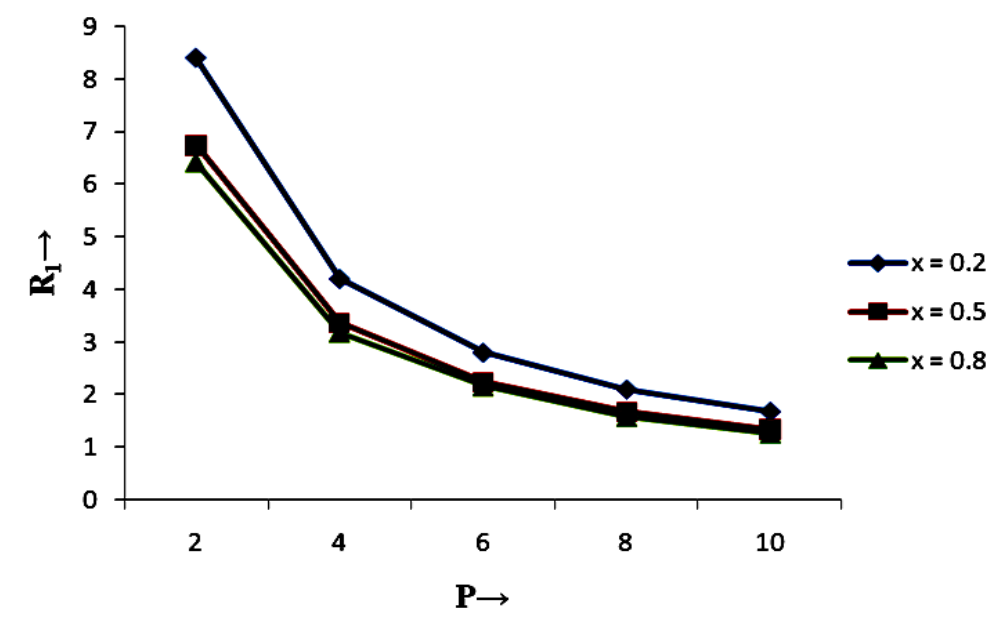

Figure 4. Variation of Rayleigh number $R_{1}$ with medium permeability $P$ for $B=3$ and $D_{A_{1}}=10$ for fixed wave numbers $x=0.2, x=0.5$ and $x=0.8$. 


\section{CONCLUSIONS}

The effect of suspended particles on thermal convection in a Rivlin-Ericksen elasticoviscous fluid heated from below in a Brinkman porous medium has been investigated. The dispersion relation, including the effects of suspended particles, Darcy number, medium permeability and viscoelasticity on the thermal convection in a Rivlin-Ericksen fluid in porous medium is derived. From the analysis, the main conclusions are as follows:

(i) For the case of stationary convection, a Rivlin-Ericksen elastico-viscous fluid behaves like an ordinary Newtonian fluid as elastico-viscous parameter $\mathrm{F}$ vanishes with $\sigma$.

(ii) The expressions for $\frac{d R_{1}}{d B}, \frac{d R_{1}}{d D_{A_{1}}}$ and $\frac{d R_{1}}{d P}$ are examined analytically and it has been found that the Darcy number has a stabilising effect, whereas the suspended particles and medium permeability have a destabilising effect on the system.

(iii) The effects of suspended particles, Darcy number and medium permeability on thermal convection in a Rivlin-Ericksen elastico-viscous fluid permeated with suspended particles in a Brinkman porous medium have been analysed.

(iv) The oscillatory modes are introduced due to the presence of viscoelasticity, suspended particles, gravity field and medium permeability, which were nonexistent in their absence.

\section{ACKNOWLEDGEMENTS}

The authors would like to thank the reviewers for their valuable comments and suggestions for the improvement of the paper.

\section{REFERENCES}

Chandrasekhar, S. (1981). Hydrodynamic and hydromagnetic stability. New York: Dover Publication.

Ingham, D. D., \& Pop, L. (1981). Transport phenomena in porous media. New York: Elsevier.

Kuznetsov, A. V., \& Nield, D. A. (2010). Thermal instability in a porous medium layer saturated by a nanofluid: Brinkman model. Transport in Porous Media, 81(3), 409-422.

Lapwood, E. R. (1948). Convection of a fluid in porous medium. Mathematical Proceedings of the Cambridge Philosophical Society, 44, 508-519.

Nield, D. A., \& Bejan, A. (2006). Convection in Porous Medium. New York: Springer.

Rana, G.C. 2011. The onset of convection in Rivlin-Ericksen fluid in a Darcy-Brinkman porous medium. International Journal of Pure and Applied Mathematical Sciences, 5, 73-80.

Rana, G. C. (2012). Thermal instability of compressible Rivlin-Ericksen elasticoviscous rotating fluid permitted with suspended dust particles in porous medium. Journal of Applied Mathematics and Mechanics, 8(4), 97-110.

Rana, G. C., \& Kumar, S. (2010). Thermal instability of Rivlin-Ericksen ElasticoViscous rotating fluid permitted with suspended particles and variable gravity field in porous medium. Studia Geotechnica et Mechanica, 32, 39-54. 
Rana, G.C., \& Thakur, R. C. (2012). A mathematical theorem on the onset of couplestress fluid permeated with suspended particles saturating a porous medium. International Journal of Multiphysics, 6, 61-72.

Rivlin, R. S., \& Ericksen, J. L. (1955). Stress-deformation relations for isotropic materials. Chemistry Biodiversity, 4(2), 323-425.

Satya Narayana, P. V., Ramireddy, G., \& Venkataramana, S. (2011). Hall current effects on free-convection MHD flow past a porous plate. International Journal of Automotive and Mechanical Engineering, 3, 350-363.

Scanlon, J. W., \& Segel, L. A. (1973). Effect of suspended particles on the onset of Be'nard convection. Physics Fluids, 16, 1573-1578.

Sharma, R. C., \& Sharma, V. (1991). Stability of stratified fluid in porous medium in the presence of suspended particles and variable magnetic field. Czech. Journal of Physics, 41, 450-462.

Sharma, R. C., \& Sunil. (1994). Thermal instability of an Oldroydian fluid with suspended particles in hydromagnetics in porous medium. Polymer-Plastics Technology and Engineering, 33(3), 323-339.

Sharma, V., Kishore, K., \& Rana, G. C. (2001). The instability of streaming RivlinEricksen fluids in porous medium. Studia Geotechnica et Mechanica, 13, 83-93.

Vafai, K., \& Hadim, H. A. (2000). Handbook of porous media. New York: M. Decker.

Wooding, R. A. (1960). Rayleigh instability of a thermal boundary layer in flow through a porous medium. Journal of Fluid Mechanics, 9, 183-192.

\section{NOMENCLATURE}

\begin{tabular}{|c|c|c|c|}
\hline$P_{l}$ & Dimensionless medium permeability & & Gravitational acceleration \\
\hline g & Gravitational acceleration vector & $\mathrm{m}$ & Mass of suspended particle \\
\hline$D_{A}$ & Modified Darcy number & $p$ & Pressure \\
\hline$K^{\prime}$ & Stokes drag coefficient & $p_{1}$ & Thermal Prandtl number \\
\hline$v$ & Velocity of fluid & $v_{d}$ & Velocity of suspended particles \\
\hline$k$ & Wave number of disturbance & & \\
\hline \multicolumn{4}{|c|}{ Greek Symbols } \\
\hline $\begin{array}{l}\beta \\
\text { me }\end{array}$ & $\begin{array}{l}\text { Adverse temperature gradient } \\
\text { um }\end{array}$ & $\tilde{\mu}$ & viscosity of \\
\hline $\boldsymbol{\rho}$ & Fluid density & $\mu$ & Fluid viscosity \\
\hline$\mu^{\prime}$ & Fluid viscoelasticity & $v$ & Kinematic viscosity \\
\hline$u^{\prime}$ & Kinematic viscoelasticity & $\varepsilon$ & Medium porosity \\
\hline$\theta$ & Perturbation in temperature & $\eta$ & Radius of suspended particles \\
\hline$\kappa$ & Thermal diffusitivity & $\alpha$ & Thermal coefficient of expansion \\
\hline
\end{tabular}

